\title{
Observation of $D^{0}$ Meson Decays to $\pi^{+} \pi^{-} \mu^{+} \mu^{-}$and $K^{+} K^{-} \mu^{+} \mu^{-}$Final States
}

\author{
R. Aaij et al. \\ (LHCb Collaboration)
}

(Received 27 July 2017; published 31 October 2017)

\begin{abstract}
The first observation of the $D^{0} \rightarrow \pi^{+} \pi^{-} \mu^{+} \mu^{-}$and $D^{0} \rightarrow K^{+} K^{-} \mu^{+} \mu^{-}$decays is reported using a sample of proton-proton collisions collected by LHCb at a center-of-mass energy of $8 \mathrm{TeV}$, and corresponding to $2 \mathrm{fb}^{-1}$ of integrated luminosity. The corresponding branching fractions are measured using as normalization the decay $D^{0} \rightarrow K^{-} \pi^{+}\left[\mu^{+} \mu^{-}\right]_{\rho^{0} / \omega}$, where the two muons are consistent with coming from the decay of a $\rho^{0}$ or $\omega$ meson. The results are $\mathcal{B}\left(D^{0} \rightarrow \pi^{+} \pi^{-} \mu^{+} \mu^{-}\right)=(9.64 \pm 0.48 \pm 0.51 \pm 0.97) \times 10^{-7}$ and $\mathcal{B}\left(D^{0} \rightarrow K^{+} K^{-} \mu^{+} \mu^{-}\right)=(1.54 \pm 0.27 \pm 0.09 \pm 0.16) \times 10^{-7}$, where the uncertainties are statistical, systematic, and due to the limited knowledge of the normalization branching fraction. The dependence of the branching fraction on the dimuon mass is also investigated.
\end{abstract}

DOI: 10.1103/PhysRevLett.119.181805

Decays of charm hadrons into final states containing dimuon pairs may proceed via the short-distance $c \rightarrow$ $u \mu^{+} \mu^{-}$flavor-changing neutral-current process, which in the standard model can only occur through electroweakloop amplitudes that are highly suppressed by the GlashowIliopoulos-Maiani mechanism [1]. If dominated by these short-distance contributions, the inclusive $D \rightarrow X \mu^{+} \mu^{-}$ branching fraction, where $X$ represents one or more hadrons, is predicted to be $\mathcal{O}\left(10^{-9}\right)$ [2] and can be greatly enhanced by the presence of new particles, making these decays interesting for searches for physics beyond the standard model. However, long-distance contributions occur through tree-level amplitudes involving intermediate resonances, such as $D \rightarrow X V\left(\rightarrow \mu^{+} \mu^{-}\right)$, where $V$ represents a $\rho^{0}, \omega$ or $\phi$ vector meson, and can increase the standard model branching fraction up to $\mathcal{O}\left(10^{-6}\right)$ [2-4]. The sensitivity to the short-distance amplitudes is greatest for dimuon masses away from resonances, though resonances populate the entire dimuon-mass spectrum due to their long tails. Additional discrimination between shortand long-distance contributions can be gained by studying angular distributions and charge-parity-conjugation asymmetries, which in scenarios beyond the standard model could be as large as $\mathcal{O}(1 \%)$ [4-9]. Decays of $D^{0}$ mesons to four-body final states (Fig. 1) are particularly interesting in this respect as they give access to a variety of angular distributions. These decays were searched for by the Fermilab E791 Collaboration and upper limits were set on the branching fractions in the range $10^{-5}-10^{-4}$ at the

*Full author list given at the end of the article.

Published by the American Physical Society under the terms of the Creative Commons Attribution 4.0 International license. Further distribution of this work must maintain attribution to the author(s) and the published article's title, journal citation, and DOI.
90\% confidence level (C.L.) [10]. More recently, a search for nonresonant $D^{0} \rightarrow \pi^{+} \pi^{-} \mu^{+} \mu^{-}$decays (the inclusion of charge-conjugate decays is implied) was performed by the LHCb Collaboration using $7 \mathrm{TeV} p$ p-collision data corresponding to $1 \mathrm{fb}^{-1}$ of integrated luminosity [11]. An upper limit of $5.5 \times 10^{-7}$ at the $90 \%$ C.L. was set on the branching fraction due to short-distance contributions, assuming a phase-space decay.

This Letter reports the first observation of $D^{0} \rightarrow$ $\pi^{+} \pi^{-} \mu^{+} \mu^{-}$and $D^{0} \rightarrow K^{+} K^{-} \mu^{+} \mu^{-}$decays using data collected by the LHCb experiment in 2012 at a center-of-mass energy $\sqrt{s}=8 \mathrm{TeV}$ and corresponding to an integrated luminosity of $2 \mathrm{fb}^{-1}$. The analysis is performed using $D^{0}$ mesons originating from $D^{*+} \rightarrow D^{0} \pi^{+}$decays, with the $D^{*+}$ meson produced directly at the primary $p p$-collision vertex (PV). The small phase space available in this decay allows for a large background rejection, which compensates for the reduction in signal yield compared to inclusively produced $D^{0}$ mesons. The signal is studied in regions of dimuon mass, $m\left(\mu^{+} \mu^{-}\right)$, defined according to the known resonances. For $D^{0} \rightarrow \pi^{+} \pi^{-} \mu^{+} \mu^{-}$decays these regions are (low-mass) $<525 \mathrm{MeV} / c^{2},(\eta)$ 525-565 MeV $/ c^{2},\left(\rho^{0} / \omega\right)$ $565-950 \mathrm{MeV} / c^{2}$, ( $\left.\phi\right)$ 950-1100 MeV/c$c^{2}$, and (highmass) $>1100 \mathrm{MeV} / c^{2}$. The same regions are considered for $D^{0} \rightarrow K^{+} K^{-} \mu^{+} \mu^{-}$decays, with the exception of the $\phi$ and high-mass regions, which are not present because of the reduced phase space, and the $\rho^{0} / \omega$ region, which extends from $565 \mathrm{MeV} / c^{2}$ up to the kinematic limit. In the regions where a signal is observed a measurement of the branching fraction is provided, otherwise $90 \%$ and $95 \%$ C.L. upper limits are set; no attempt is made to distinguish between the short- and long-distance contributions in each dimuon-mass region. The branching fraction is measured using as a normalization the $D^{0} \rightarrow K^{-} \pi^{+}\left[\mu^{+} \mu^{-}\right]_{\rho^{0} / \omega}$ decay in the dimuon-mass range $675-875 \mathrm{MeV} / c^{2}$, where the contribution from the $\rho^{0} / \omega \rightarrow \mu^{+} \mu^{-}$decay is dominant. The 


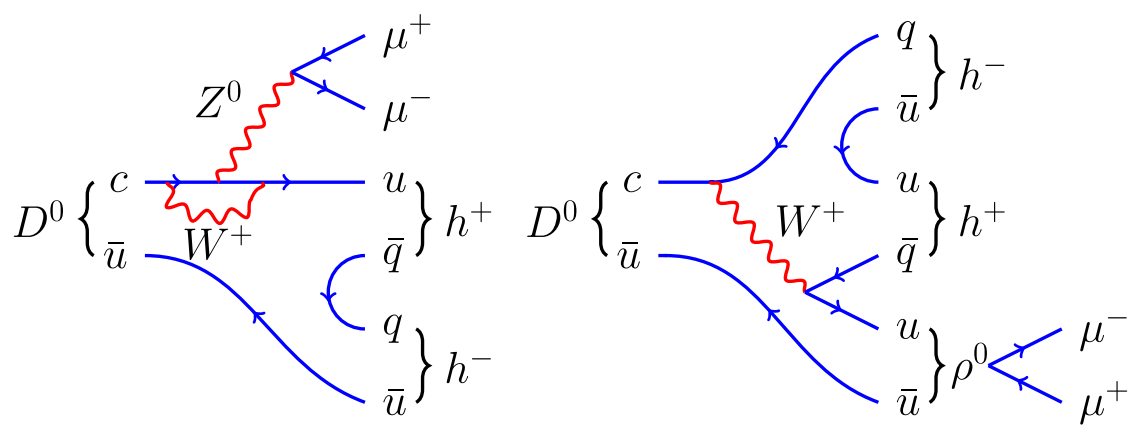

FIG. 1. Example diagrams describing the (left) short- and (right) long-distance contributions to $D^{0} \rightarrow h^{+} h^{-} \mu^{+} \mu^{-}$decays, where $q=d, s$ and $h=\pi, K$.

$D^{0} \rightarrow K^{-} \pi^{+}\left[\mu^{+} \mu^{-}\right]_{\rho^{0} / \omega}$ branching fraction was recently measured to be $(4.17 \pm 0.42) \times 10^{-6}[12]$ and provides a more precise normalization than that used in the previous LHCb search [11].

The $\mathrm{LHCb}$ detector is a single-arm forward spectrometer $[13,14]$. It includes a high-precision tracking system consisting of a silicon-strip vertex detector surrounding the $p p$-interaction region, a large-area silicon-strip detector located upstream of a dipole magnet with a bending power of about $4 \mathrm{Tm}$, and three stations of silicon-strip detectors and straw drift tubes placed downstream of the magnet. Particle identification is provided by two ring-imaging Cherenkov detectors, an electromagnetic and a hadronic calorimeter, and a muon system composed of alternating layers of iron and multiwire proportional chambers.

Events are selected online by a trigger that consists of a hardware stage, which is based on information from the calorimeter and muon systems, followed by a software stage, which applies a full event reconstruction [15]. The hardware trigger requires the presence in the event of a muon with transverse momentum, $p_{\mathrm{T}}$, exceeding $1.76 \mathrm{GeV} / c$. A first stage of the software trigger selects events with a charged particle of $p_{\mathrm{T}}>1.6 \mathrm{GeV} / c$ and significant impact parameter, defined as the minimum distance of the particle trajectory from any $\mathrm{PV}$, or alternatively with $p_{\mathrm{T}}>1 \mathrm{GeV} / c$ if the particle has associated hits in the muon system. In a second stage of the software trigger, dedicated algorithms select candidate $D^{0} \rightarrow h^{+} h^{(\prime)}-\mu^{+} \mu^{-}$decays, where $h$ is either a kaon or a pion, from combinations of four tracks, each having momentum $p>3 \mathrm{GeV} / c$ and $p_{\mathrm{T}}>$ $0.5 \mathrm{GeV} / c$, that form a secondary vertex separated from any PV. Two oppositely charged particles are required to leave hits in the muon system and the scalar sum of their $p_{\mathrm{T}}$ is required to exceed $3 \mathrm{GeV} / c$. The mass of the $D^{0}$ candidate, $m\left(D^{0}\right)$, has to be in the range $1800-1940 \mathrm{MeV} / c^{2}$ and its momentum must be aligned with the vector connecting the primary and secondary vertices.

In the offline analysis, $D^{0}$ candidates satisfying the trigger requirements are further selected through particleidentification criteria placed on their decay products. They are then combined with a charged particle originating from the same PV and having $p_{\mathrm{T}}>120 \mathrm{MeV} / c$, to form a $D^{*+} \rightarrow D^{0} \pi^{+}$candidate. When more than one $\mathrm{PV}$ is reconstructed, the one with respect to which the $D^{0}$ candidate has the lowest impact-parameter significance is chosen. The vertex formed by the $D^{0}$ and $\pi^{+}$mesons is constrained to coincide with the PV and the difference between the $D^{*+}$ and $D^{0}$ masses, $\Delta m$, is required to be in the range $144.5-146.5 \mathrm{MeV} / c^{2}$. A multivariate selection based on a boosted decision tree (BDT) [16,17] with gradient boosting [18] is then used to suppress background from combinations of unrelated charged particles. The features used by the BDT to discriminate signal from this combinatorial background are as follows: the momentum and transverse momentum of the pion from the $D^{*+}$ decay, the smallest impact parameter of the $D^{0}$ decay products with respect to the PV, the angle between the $D^{0}$ momentum and the vector connecting the primary and secondary vertices, the quality of the secondary vertex, its separation from the PV, and its separation from any other track not forming the $D^{*+}$ candidate. The BDT is trained separately for $D^{0} \rightarrow \pi^{+} \pi^{-} \mu^{+} \mu^{-}$and $D^{0} \rightarrow K^{+} K^{-} \mu^{+} \mu^{-}$decays, due to their different kinematic properties, using simulated $[19,20]$ decays as signal and data candidates with $m\left(D^{0}\right)$ between 1890 and $1940 \mathrm{MeV} / \mathrm{c}^{2}$ as background. To minimize biases on the background classification, the training samples are further randomly split into two disjoint subsamples. The classifier trained on one sample is applied to the other, and vice versa. Another source of background is due to the hadronic four-body decays $D^{0} \rightarrow \pi^{+} \pi^{-} \pi^{+} \pi^{-}$ and $D^{0} \rightarrow K^{+} K^{-} \pi^{+} \pi^{-}$, where two pions are misidentified as muons. The misidentification occurs mainly when the pions decay in flight into a muon and an undetected neutrino. Although this process is relatively rare, the large branching fractions of the hadronic modes produce a peaking background which is partially suppressed by a multivariate muon-identification discriminant that combines the information from the Cherenkov detectors, the calorimeters and the muon chambers. Thresholds on the BDT response and on the muon-identification discriminant are optimized simultaneously by maximizing $\epsilon_{h^{+} h^{-} \mu^{+} \mu^{-}} /\left(5 / 2+\sqrt{N_{\mathrm{bkg}}}\right)$ [21], where $\epsilon_{h^{+} h^{-} \mu^{+} \mu^{-}}$is the 
signal efficiency and $N_{\mathrm{bkg}}$ is the sum of the expected combinatorial and peaking background yields in the $m\left(D^{0}\right)$ range $1830-1900 \mathrm{MeV} / c^{2}$ (signal region). Candidate $D^{0} \rightarrow K^{-} \pi^{+}\left[\mu^{+} \mu^{-}\right]_{\rho^{0} / \omega}$ decays are selected using the response of the BDT trained on the $D^{0} \rightarrow \pi^{+} \pi^{-} \mu^{+} \mu^{-}$ signal, when they are used as normalization for the measurement of $\mathcal{B}\left(D^{0} \rightarrow \pi^{+} \pi^{-} \mu^{+} \mu^{-}\right)$, and that of the BDT trained on the $D^{0} \rightarrow K^{+} K^{-} \mu^{+} \mu^{-}$signal, when used as normalization for $\mathcal{B}\left(D^{0} \rightarrow K^{+} K^{-} \mu^{+} \mu^{-}\right)$. After selection, a few percent of the events contain multiple candidates, of which only one is randomly selected if they share at least one final-state particle. To avoid potential biases on the measured quantities, candidate decays in the $m\left(D^{0}\right)$ signal region are examined only after the analysis procedure has been finalized, with the exception of those populating the $\rho^{0} / \omega$ and $\phi$ dimuon-mass regions of the $D^{0} \rightarrow \pi^{+} \pi^{-} \mu^{+} \mu^{-}$ sample.

The $D^{0} \rightarrow \pi^{+} \pi^{-} \mu^{+} \mu^{-}$and $D^{0} \rightarrow K^{+} K^{-} \mu^{+} \mu^{-}$signal yields are measured with unbinned extended maximum likelihood fits to the $m\left(D^{0}\right)$ distributions (Figs. 2 and 3, respectively). The fits include three components: signal, peaking background from misidentified hadronic decays, and combinatorial background. The signal is described with a Johnson's $S_{U}$ distribution [22] with parameters determined from simulation. To account for known differences between data and simulation, the means and

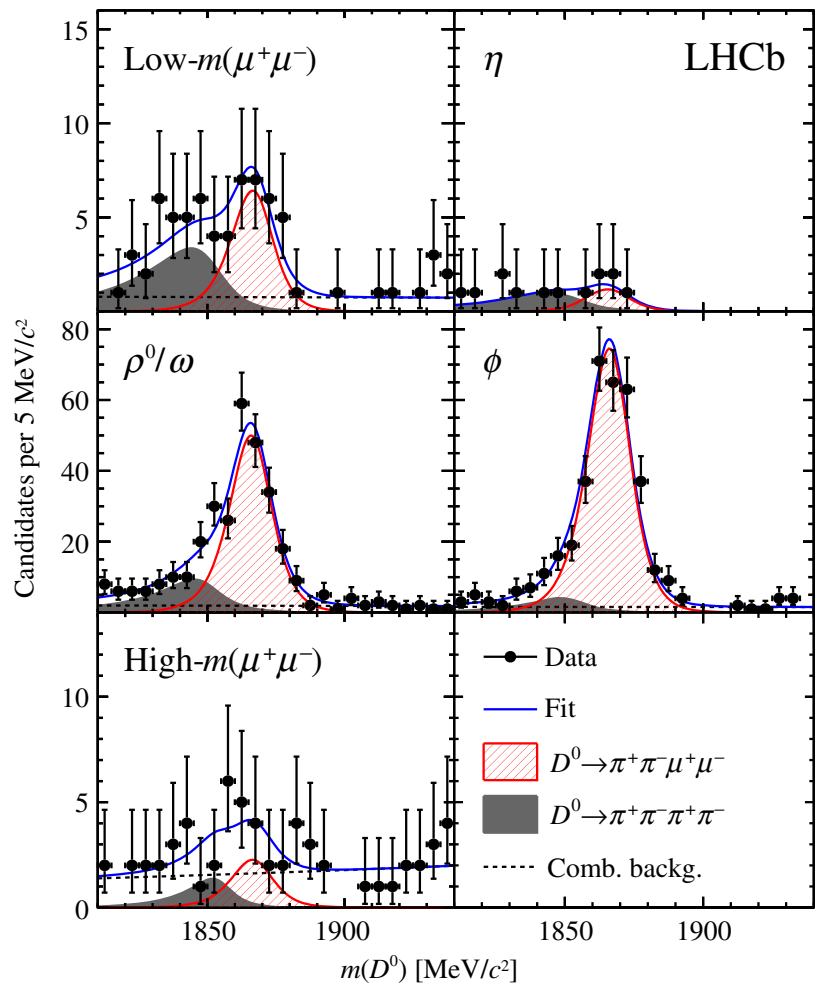

FIG. 2. Distributions of $m\left(D^{0}\right)$ for the $D^{0} \rightarrow \pi^{+} \pi^{-} \mu^{+} \mu^{-}$ candidates in the low- $m\left(\mu^{+} \mu^{-}\right), \eta, \rho^{0} / \omega, \phi$ and high- $m\left(\mu^{+} \mu^{-}\right)$ regions. Fit projections are overlaid. widths of the signal distributions are corrected using scaling factors adjusted on the normalization channel. The mass shape of the peaking background is determined using separate data samples of $D^{0} \rightarrow h^{+} h^{(\prime)} \pi^{+} \pi^{-}$decays where the $D^{0}$ mass is calculated assigning the muon-mass hypothesis to two oppositely charged pions. The combinatorial background is described by an exponential function, which is determined from data candidates with $\Delta m$ between 150 and $160 \mathrm{MeV} / c^{2}$ that fail the BDT selection. All shape parameters are fixed and only the yields are allowed to vary in the fits, which are performed separately in each $m\left(\mu^{+} \mu^{-}\right)$range.

The resulting signal yields are reported in Table I. No fit is performed in the $\eta$ region of the $D^{0} \rightarrow K^{+} K^{-} \mu^{+} \mu^{-}$ dimuon-mass spectrum, where only two candidates are observed. An excess of candidates with respect to the background-only hypothesis is seen with a significance above three standard deviations in all dimuon-mass ranges with the exception of the $\eta$ region of both decays and the high- $m\left(\mu^{+} \mu^{-}\right)$region of $D^{0} \rightarrow \pi^{+} \pi^{-} \mu^{+} \mu^{-}$. The significances are determined from the change in likelihood from fits with and without the signal component.

The signal yields, $N_{h^{+} h^{-} \mu^{+} \mu^{-}}$, in each $m\left(\mu^{+} \mu^{-}\right)$range $i$ are converted into branching fractions using

$$
\begin{aligned}
\mathcal{B}^{i}\left(D^{0} \rightarrow h^{+} h^{-} \mu^{+} \mu^{-}\right) \\
=\frac{N_{h^{+} h^{-} \mu^{+} \mu^{-}} \mathcal{B}\left(D^{0} \rightarrow K^{-} \pi^{+}\left[\mu^{+} \mu^{-}\right]_{\rho^{0} / \omega}\right)}{R_{\epsilon}^{i} N_{K^{-} \pi^{+} \mu^{+} \mu^{-}}},
\end{aligned}
$$

where $N_{K^{-} \pi^{+} \mu^{+} \mu^{-}}$is the yield of the normalization mode, which is determined to be $1971 \pm 51(1806 \pm 48)$ after the selection optimized for $D^{0} \rightarrow \pi^{+} \pi^{-} \mu^{+} \mu^{-}$

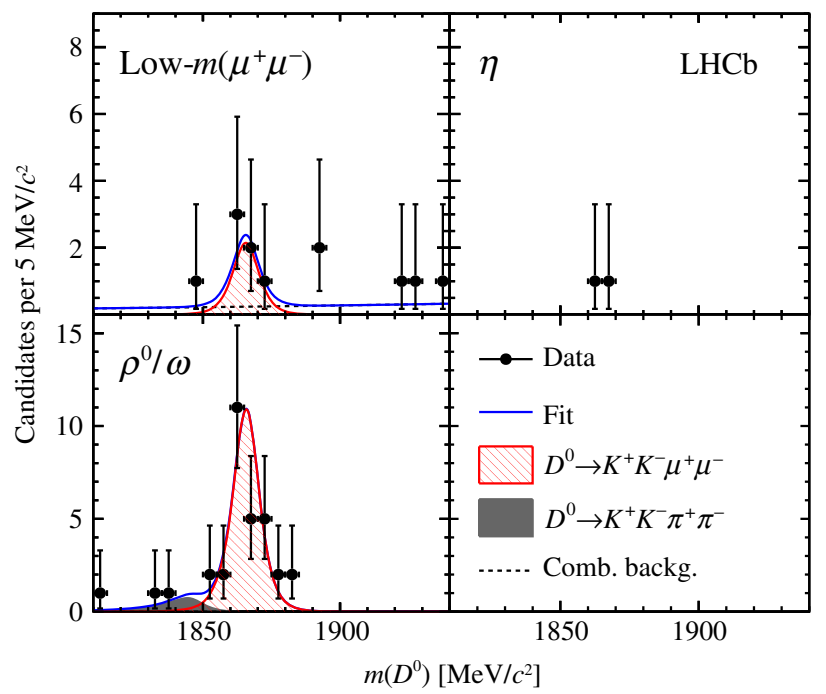

FIG. 3. Distributions of $m\left(D^{0}\right)$ for the $D^{0} \rightarrow K^{+} K^{-} \mu^{+} \mu^{-}$ candidates in the low- $m\left(\mu^{+} \mu^{-}\right), \eta$ and $\rho^{0} / \omega$ regions. Fit projections are overlaid. No fit is performed in the $\eta$ region, where only two candidates are observed. 
TABLE I. Yields of (top) $D^{0} \rightarrow \pi^{+} \pi^{-} \mu^{+} \mu^{-}$and (bottom) $D^{0} \rightarrow K^{+} K^{-} \mu^{+} \mu^{-}$signal decays, their significance with respect to the background-only hypothesis, and ratio of efficiencies between signal and normalization decays $\left(R_{\epsilon}^{i}\right)$ for each dimuon-mass region. The yield and the significance $(\mathcal{S})$ are not reported for the $\eta$ region of $D^{0} \rightarrow K^{+} K^{-} \mu^{+} \mu^{-}$, where only two candidates are observed.

\begin{tabular}{|c|c|c|c|c|}
\hline$m\left(\mu^{+} \mu^{-}\right)$region & {$\left[\mathrm{MeV} / c^{2}\right]$} & Yield & $\mathcal{S}$ & $R_{\epsilon}^{i}$ \\
\hline \multicolumn{5}{|c|}{$D^{0} \rightarrow \pi^{+} \pi^{-} \mu^{+} \mu^{-}$} \\
\hline Low mass & $<525$ & $27 \pm 6$ & $5.4 \sigma$ & $0.73 \pm 0.04$ \\
\hline$\eta$ & $525-565$ & $5 \pm 3$ & $2.5 \sigma$ & $0.84 \pm 0.07$ \\
\hline$\rho^{0} / \omega$ & $565-950$ & $208 \pm 17$ & $18 \sigma$ & $1.08 \pm 0.05$ \\
\hline$\phi$ & $950-1100$ & $312 \pm 20$ & $23 \sigma$ & $1.45 \pm 0.07$ \\
\hline High mass & $>1100$ & $9 \pm 6$ & $1.6 \sigma$ & $1.5 \pm 0.1$ \\
\hline \multicolumn{5}{|c|}{$D^{0} \rightarrow K^{+} K^{-} \mu^{+} \mu^{-}$} \\
\hline Low mass & $<525$ & $5 \pm 3$ & $3.1 \sigma$ & $0.49 \pm 0.03$ \\
\hline$\eta$ & $525-565$ & $\cdots$ & $\cdots$ & $0.53 \pm 0.04$ \\
\hline$\rho^{0} / \omega$ & $>565$ & $29 \pm 5$ & $8.1 \sigma$ & $0.55 \pm 0.03$ \\
\hline
\end{tabular}

$\left(D^{0} \rightarrow K^{+} K^{-} \mu^{+} \mu^{-}\right)$decays. The ratios of geometrical acceptances, and reconstruction and selection efficiencies of the signal relative to the normalization decays, $R_{\epsilon}^{i}=\epsilon_{h^{+} h^{-} \mu^{+} \mu^{-}}^{i} / \epsilon_{K^{-} \pi^{+} \mu^{+} \mu^{-}}$, are reported in Table I. They are determined using simulated events and corrected to account for known differences between data and simulation. In particular, particle-identification and hardwaretrigger efficiencies are measured from control channels in data.

Systematic uncertainties affect the determination of the signal and normalization yields, and of the efficiency ratio. For the determination of the yields, effects due to uncertainties on the $m\left(D^{0}\right)$ shapes are investigated. A possible dependence on the decay mode or on the $m\left(\mu^{+} \mu^{-}\right)$range of the scaling factors, used to account for data-simulation differences, is quantified using fits to the $D^{0} \rightarrow$ $\pi^{+} \pi^{-}\left[\mu^{+} \mu^{-}\right]_{\phi}$ and $D^{0} \rightarrow \pi^{+} \pi^{-}\left[\mu^{+} \mu^{-}\right]_{\rho^{0} / \omega}$ data and is found to be negligible. To assess the impact of $\pi \rightarrow \mu \nu$ decays in flight, alternative shapes are tested for the $D^{0} \rightarrow$ $h^{+} h^{(\prime)}{ }^{+} \pi^{+} \pi^{-}$background by changing the muonidentification and the $p_{\mathrm{T}}$ requirements on the misidentified pions. The largest observed variation in the ratio of $D^{0} \rightarrow$ $\pi^{+} \pi^{-}\left[\mu^{+} \mu^{-}\right]_{\phi}$ to $D^{0} \rightarrow K^{-} \pi^{+}\left[\mu^{+} \mu^{-}\right]_{\rho^{0} / \omega}$ yields $(1.4 \%)$ is assigned as a systematic uncertainty for both $h^{+} h^{-} \mu^{+} \mu^{-}$ modes and all dimuon-mass ranges. Changes in the shape of the peaking background introduced by the different trigger requirements used to select the hadronic decays are negligible. The fit to the data is repeated using alternative descriptions of the combinatorial background, determined from data sidebands defined by different BDT and $\Delta m$ requirements, and results in negligible variations of the signal and normalization yields.

Systematic uncertainties affecting the efficiency ratio include data-simulation differences that are not accounted for and limitations in the data-driven methods used to determine the particle-identification and trigger efficiencies. The signal decays are simulated with an incoherent sum of resonant and nonresonant dimuon and dihadron components, while the resonant structure in data is unknown. A systematic uncertainty of $3.4 \%$ on the signal efficiency is determined by varying the relative fractions of these components. A systematic uncertainty of $1.0 \%$ on the efficiency ratio is assigned due to the criteria used in simulation to match the reconstructed and generated particles. Muon- and hadron-identification efficiencies are determined from data by weighting the kinematic properties of the calibration samples to match those of the signal samples. Variations of the choice of the binning scheme used in the weighting procedure change the efficiency ratio by up to $0.8 \%$, which is taken as systematic uncertainty. The datadriven method that evaluates the hardware-trigger efficiency ratio is validated in simulation to be unbiased within $1.3 \%$, which is assigned as a systematic uncertainty. The efficiencies of the BDT requirement for the simulated normalization and $D^{0} \rightarrow \pi^{+} \pi^{-}\left[\mu^{+} \mu^{-}\right]_{\phi}$ decays are compared to those obtained from background-subtracted data. A difference in the efficiency ratio of $1.3 \%$ is observed and assigned as systematic uncertainty.

Finally, the statistical uncertainty on the normalization yield introduces a relative uncertainty of $2.6 \%(2.7 \%)$, which is propagated to the systematic uncertainty on the $D^{0} \rightarrow \pi^{+} \pi^{-} \mu^{+} \mu^{-}\left(D^{0} \rightarrow K^{+} K^{-} \mu^{+} \mu^{-}\right)$branching fractions.

Table II reports the measured values and upper limits on the $D^{0} \rightarrow \pi^{+} \pi^{-} \mu^{+} \mu^{-}$and $D^{0} \rightarrow K^{+} K^{-} \mu^{+} \mu^{-}$branching fractions in the various ranges of $m\left(\mu^{+} \mu^{-}\right)$, where the first uncertainty accounts for the statistical component, the second for the systematic, and the third corresponds to the $10 \%$ relative uncertainty on $\mathcal{B}\left(D^{0} \rightarrow K^{-} \pi^{+}\left[\mu^{+} \mu^{-}\right]_{\rho^{0} / \omega}\right)$ [12]. The upper limits are derived using a frequentist approach based on a likelihood-ratio ordering method that includes the effects due to the systematic uncertainties $[23,24]$. For the $\eta$ region of $D^{0} \rightarrow K^{+} K^{-} \mu^{+} \mu^{-}$, where no fit is performed, the limit is calculated assuming two signal 
TABLE II. Branching fractions of (top) $D^{0} \rightarrow \pi^{+} \pi^{-} \mu^{+} \mu^{-}$and (bottom) $D^{0} \rightarrow K^{+} K^{-} \mu^{+} \mu^{-}$decays in different ranges of dimuon mass, where the uncertainties are statistical, systematic, and due to the limited knowledge of the normalization branching fraction. The reported upper limits correspond to $90 \%$ (95\%) C.L. The correlations between the various dimuon-mass ranges are reported in the Supplemental Material [25].

\begin{tabular}{|c|c|c|}
\hline$m\left(\mu^{+} \mu^{-}\right)$region & {$\left[\mathrm{MeV} / c^{2}\right]$} & $\mathcal{B}\left[10^{-8}\right]$ \\
\hline \multicolumn{3}{|c|}{$D^{0} \rightarrow \pi^{+} \pi^{-} \mu^{+} \mu^{-}$} \\
\hline Low mass & $<525$ & $7.8 \pm 1.9 \pm 0.5 \pm 0.8$ \\
\hline$\eta$ & $525-565$ & $<2.4(2.8)$ \\
\hline$\rho^{0} / \omega$ & $565-950$ & $40.6 \pm 3.3 \pm 2.1 \pm 4.1$ \\
\hline$\phi$ & $950-1100$ & $45.4 \pm 2.9 \pm 2.5 \pm 4.5$ \\
\hline High mass & $>1100$ & $<2.8(3.3)$ \\
\hline \multicolumn{3}{|c|}{$D^{0} \rightarrow K^{+} K^{-} \mu^{+} \mu^{-}$} \\
\hline Low mass & $<525$ & $2.6 \pm 1.2 \pm 0.2 \pm 0.3$ \\
\hline$\eta$ & $525-565$ & $<0.7(0.8)$ \\
\hline$\rho^{0} / \omega$ & $>565$ & $12.0 \pm 2.3 \pm 0.7 \pm 1.2$ \\
\hline
\end{tabular}

candidates and zero background. Integrating over dimuon mass, and accounting for correlations [25], the total branching fractions are measured to be

$$
\begin{aligned}
& \mathcal{B}\left(D^{0} \rightarrow \pi^{+} \pi^{-} \mu^{+} \mu^{-}\right) \\
& \quad=(9.64 \pm 0.48 \pm 0.51 \pm 0.97) \times 10^{-7}, \\
& \mathcal{B}\left(D^{0} \rightarrow K^{+} K^{-} \mu^{+} \mu^{-}\right) \\
& \quad=(1.54 \pm 0.27 \pm 0.09 \pm 0.16) \times 10^{-7} .
\end{aligned}
$$

The two results have a correlation of 0.497 and are consistent with the standard model expectations [4].

In summary, a study of the $D^{0} \rightarrow \pi^{+} \pi^{-} \mu^{+} \mu^{-}$and $D^{0} \rightarrow$ $K^{+} K^{-} \mu^{+} \mu^{-}$decays is performed in ranges of the dimuon mass using $p p$ collisions collected by the LHCb experiment at $\sqrt{s}=8 \mathrm{TeV}$. Significant signal yields are observed for the first time in several dimuon-mass ranges for both decays; the corresponding branching fractions are measured and found to be consistent with the standard model expectations [4]. For the dimuon-mass regions where no significant signal is observed, upper limits at $90 \%$ and $95 \%$ C.L. are set on the branching fraction. The total branching fractions are measured to be $\mathcal{B}\left(D^{0} \rightarrow \pi^{+} \pi^{-} \mu^{+} \mu^{-}\right)=(9.64 \pm 0.48 \pm 0.51 \pm$ $0.97) \times 10^{-7} \quad$ and $\quad \mathcal{B}\left(D^{0} \rightarrow K^{+} K^{-} \mu^{+} \mu^{-}\right)=(1.54 \pm 0.27 \pm$ $0.09 \pm 0.16) \times 10^{-7}$, where the uncertainties are statistical, systematic, and due to the limited knowledge of the normalization branching fraction. These are the rarest charm-hadron decays ever observed and are expected to provide better sensitivity to short-distance flavor-changing neutral-current contributions to these decays.

We express our gratitude to our colleagues in the CERN accelerator departments for the excellent performance of the LHC. We thank the technical and administrative staff at the LHCb institutes. We acknowledge support from CERN and from the national agencies: CAPES, CNPq, FAPERJ and FINEP (Brazil); MOST and NSFC (China); CNRS/ IN2P3 (France); BMBF, DFG and MPG (Germany); INFN (Italy); NWO (Netherlands); MNiSW and NCN (Poland); MEN/IFA (Romania); MinES and FASO (Russia); MinECo (Spain); SNSF and SER (Switzerland); NASU (Ukraine); STFC (United Kingdom); NSF (USA). We acknowledge the computing resources that are provided by CERN, IN2P3 (France), KIT and DESY (Germany), INFN (Italy), SURF (Netherlands), PIC (Spain), GridPP (United Kingdom), RRCKI and Yandex LLC (Russia), CSCS (Switzerland), IFIN-HH (Romania), CBPF (Brazil), PL-GRID (Poland) and OSC (USA). We are indebted to the communities behind the multiple open source software packages on which we depend. Individual groups or members have received support from AvH Foundation (Germany), EPLANET, Marie Skłodowska-Curie Actions and ERC (European Union), Conseil Général de Haute-Savoie, Labex ENIGMASS and OCEVU, Région Auvergne (France), RFBR and Yandex LLC (Russia), GVA, XuntaGal and GENCAT (Spain), Herchel Smith Fund, The Royal Society, Royal Commission for the Exhibition of 1851 and the Leverhulme Trust (United Kingdom).

[1] S. L. Glashow, J. Iliopoulos, and L. Maiani, Weak interactions with lepton-hadron symmetry, Phys. Rev. D 2, 1285 (1970).

[2] A. Paul, I. I. Bigi, and S. Recksiegel, On $D \rightarrow X_{u} \ell^{+} \ell^{-}$ within the standard model and frameworks like the littlest Higgs model with T parity, Phys. Rev. D 83, 114006 (2011).

[3] S. Fajfer, N. Košnik, and S. Prelovšek, Updated constraints on new physics in rare charm decays, Phys. Rev. D 76, 074010 (2007).

[4] L. Cappiello, O. Cata, and G. D'Ambrosio, Standard model prediction and new physics tests for $D^{0} \rightarrow h_{1}^{+} h_{2}^{-} \ell^{+} \ell^{-}$ ( $h=\pi, K ; \ell=e, \mu)$, J. High Energy Phys. 04 (2013) 135.

[5] I. I. Bigi and A. Paul, On CP asymmetries in two-, threeand four-body $D$ decays, J. High Energy Phys. 03 (2012) 021.

[6] A. Paul, A. de la Puente, and I. I. Bigi, Manifestations of warped extra dimension in rare charm decays and asymmetries, Phys. Rev. D 90, 014035 (2014).

[7] S. Fajfer and N. Košnik, Prospects of discovering new physics in rare charm decays, Eur. Phys. J. C 75, 567 (2015).

[8] S. Fajfer and S. Prelovšek, Effects of littlest Higgs model in rare D meson decays, Phys. Rev. D 73, 054026 (2006).

[9] S. de Boer and G. Hiller, Flavor and new physics opportunities with rare charm decays into leptons, Phys. Rev. D 93, 074001 (2016).

[10] E. M. Aitala et al. (E791 Collaboration), Search for Rare and Forbidden Charm Meson Decays $D^{0} \rightarrow V \ell^{+} \ell^{-}$and hhel, Phys. Rev. Lett. 86, 3969 (2001).

[11] R. Aaij et al. (LHCb Collaboration), Search for the decay $D^{0} \rightarrow \pi^{+} \pi^{-} \mu^{+} \mu^{-}$, Phys. Lett. B 728, 234 (2014). 
[12] R. Aaij et al. (LHCb Collaboration), First observation of the decay $D^{0} \rightarrow K^{-} \pi^{+} \mu^{+} \mu^{-}$in the $\rho^{0}-\omega$ region of the dimuon mass spectrum, Phys. Lett. B 757, 558 (2016).

[13] A. A. Alves Jr. et al. (LHCb Collaboration), The LHCb detector at the LHC, J. Instrum. 3, S08005 (2008).

[14] R. Aaij et al. (LHCb Collaboration), LHCb detector performance, Int. J. Mod. Phys. A 30, 1530022 (2015).

[15] R. Aaij et al. (LHCb Collaboration), The LHCb trigger and its performance in 2011, J. Instrum. 8, P04022 (2013).

[16] L. Breiman, J. H. Friedman, R. A. Olshen, and C. J. Stone, Classification and Regression Trees (Wadsworth International Group, Belmont, CA, 1984).

[17] B. P. Roe, H.-J. Yang, J. Zhu, Y. Liu, I. Stancu, and G. McGregor, Boosted decision trees as an alternative to artificial neural networks for particle identification, Nucl. Instrum. Methods Phys. Res., Sect. A 543, 577 (2005).

[18] A. Hoecker et al., TMVA-Toolkit for multivariate data analysis, Proc. Sci., ACAT (2007) 040.

[19] M. Clemencic, G. Corti, S. Easo, C. R. Jones, S. Miglioranzi, M. Pappagallo, and P. Robbe, The LHCb simulation application, Gauss: Design, evolution and experience, J. Phys. Conf. Ser. 331, 032023 (2011).

[20] I. Belyaev et al., Handling of the generation of primary events in Gauss, the LHCb simulation framework, J. Phys. Conf. Ser. 331, 032047 (2011).

[21] G. Punzi, Sensitivity of searches for new signals and its optimization, edited by L. Lyons, R. Mount, and R. Reitmeyer, eConf. C030908, 79 (2003).

[22] N. L. Johnson, Systems of frequency curves generated by methods of translation, Biometrika 36, 149 (1949).

[23] A. L. Read, Presentation of search results: The $\mathrm{CL}_{\mathrm{s}}$ technique, J. Phys. G 28, 2693 (2002).

[24] T. Junk, Confidence level computation for combining searches with small statistics, Nucl. Instrum. Methods Phys. Res., Sect. A 434, 435 (1999).

[25] See Supplemental Material at http://link.aps.org/ supplemental/10.1103/PhysRevLett.119.181805 for the correlations between the branching fractions of $D^{0} \rightarrow$ $\pi^{+} \pi^{-} \mu^{+} \mu^{-}$and $D^{0} \rightarrow K^{+} K^{-} \mu^{+} \mu^{-}$decays in the dimuonmass regions.

R. Aaij, ${ }^{40}$ B. Adeva,${ }^{39}$ M. Adinolfi, ${ }^{48}$ Z. Ajaltouni, ${ }^{5}$ S. Akar,${ }^{59}$ J. Albrecht,${ }^{10}$ F. Alessio, ${ }^{40}$ M. Alexander, ${ }^{53}$ A. Alfonso Albero, ${ }^{38} \mathrm{~S}$. Ali, ${ }^{43}$ G. Alkhazov, ${ }^{31}$ P. Alvarez Cartelle, ${ }^{55}$ A. A. Alves Jr., ${ }^{59}$ S. Amato, ${ }^{2}$ S. Amerio, ${ }^{23}$ Y. Amhis, ${ }^{7}$ L. An, ${ }^{3}$ L. Anderlini, ${ }^{18}$ G. Andreassi, ${ }^{41}$ M. Andreotti, ${ }^{17, a}$ J. E. Andrews,${ }^{60}$ R. B. Appleby, ${ }^{56}$ F. Archilli, ${ }^{43}$ P. d'Argent, ${ }^{12}$ J. Arnau Romeu, ${ }^{6}$ A. Artamonov, ${ }^{37}$ M. Artuso, ${ }^{61}$ E. Aslanides, ${ }^{6}$ G. Auriemma, ${ }^{26}$ M. Baalouch, ${ }^{5}$ I. Babuschkin, ${ }^{56}$ S. Bachmann, ${ }^{12}$ J. J. Back, ${ }^{50}$ A. Badalov, ${ }^{38, b}$ C. Baesso, ${ }^{62}$ S. Baker, ${ }^{55}$ V. Balagura, ${ }^{7, \mathrm{c}}$ W. Baldini, ${ }^{17}$ A. Baranov, ${ }^{35}$ R. J. Barlow, ${ }^{56}$ C. Barschel,${ }^{40}$ S. Barsuk, ${ }^{7}$ W. Barter ${ }^{56}$ F. Baryshnikov, ${ }^{32}$ V. Batozskaya, ${ }^{29}$ V. Battista ${ }^{41}$ A. Bay, ${ }^{41}$ L. Beaucourt, ${ }^{4}$ J. Beddow ${ }^{53}$ F. Bedeschi, ${ }^{24}$ I. Bediaga, ${ }^{1}$ A. Beiter ${ }^{61}$ L. J. Bel, ${ }^{43}$ N. Beliy, ${ }^{63}$ V. Bellee,${ }^{41}$ N. Belloli, ${ }^{21, d}$ K. Belous, ${ }^{37}$ I. Belyaev, ${ }^{32}$ E. Ben-Haim, ${ }^{8}$ G. Bencivenni, ${ }^{19}$ S. Benson, ${ }^{43}$ S. Beranek, ${ }^{9}$ A. Berezhnoy, ${ }^{33}$ R. Bernet, ${ }^{42}$ D. Berninghoff, ${ }^{12}$ E. Bertholet, ${ }^{8}$ A. Bertolin, ${ }^{23}$ C. Betancourt, ${ }^{42}$ F. Betti, ${ }^{15}$ M.-O. Bettler, ${ }^{40}$ M. van Beuzekom, ${ }^{43}$ Ia. Bezshyiko, ${ }^{42}$ S. Bifani, ${ }^{47}$ P. Billoir, ${ }^{8}$ A. Birnkraut, ${ }^{10}$ A. Bitadze,${ }^{56}$ A. Bizzeti, ${ }^{18, e}$ M. Bjørn, ${ }^{57}$ T. Blake,${ }^{50}$ F. Blanc, ${ }^{41}$ J. Blouw, ${ }^{11}$ S. Blusk, ${ }^{61}$ V. Bocci, ${ }^{26}$ T. Boettcher, ${ }^{58}$ A. Bondar, ${ }^{36, f}$ N. Bondar, ${ }^{31}$ W. Bonivento, ${ }^{16}$ I. Bordyuzhin, ${ }^{32}$ A. Borgheresi, ${ }^{21, d}$ S. Borghi, ${ }^{56}$ M. Borisyak, ${ }^{35}$ M. Borsato, ${ }^{39}$ F. Bossu, ${ }^{7}$ M. Boubdir, ${ }^{9}$ T. J. V. Bowcock,${ }^{54}$ E. Bowen, ${ }^{42}$ C. Bozzi,${ }^{17,40}$ S. Braun, ${ }^{12}$ T. Britton, ${ }^{61}$ J. Brodzicka, ${ }^{27}$ D. Brundu, ${ }^{16}$ E. Buchanan, ${ }^{48}$ C. Burr, ${ }^{56}$ A. Bursche, ${ }^{16, g}$ J. Buytaert, ${ }^{40}$ W. Byczynski, ${ }^{40}$ S. Cadeddu, ${ }^{16}$ H. Cai,${ }^{64}$ R. Calabrese, ${ }^{17, a}$ R. Calladine, ${ }^{47}$ M. Calvi, ${ }^{21, d}$ M. Calvo Gomez, ${ }^{38, b}$ A. Camboni, ${ }^{38, b}$ P. Campana, ${ }^{19}$ D. H. Campora Perez, ${ }^{40}$ L. Capriotti, ${ }^{56}$ A. Carbone, ${ }^{15, h}$ G. Carboni, ${ }^{25, i}$ R. Cardinale, ${ }^{20, j}$ A. Cardini, ${ }^{16}$ P. Carniti, ${ }^{21, d}$ L. Carson, ${ }^{52}$ K. Carvalho Akiba, ${ }^{2}$ G. Casse,${ }^{54}$ L. Cassina, ${ }^{21}$ L. Castillo Garcia,${ }^{41}$ M. Cattaneo, ${ }^{40}$ G. Cavallero, ${ }^{20,40, j}$ R. Cenci, ${ }^{24, k}$ D. Chamont, ${ }^{7}$ M. Charles, ${ }^{8}$ Ph. Charpentier ${ }^{40}$ G. Chatzikonstantinidis, ${ }^{47}$ M. Chefdeville, ${ }^{4}$

S. Chen ${ }^{56}$ S. F. Cheung, ${ }^{57}$ S.-G. Chitic, ${ }^{40}$ V. Chobanova, ${ }^{39}$ M. Chrzaszcz,${ }^{42,27}$ A. Chubykin, ${ }^{31}$ P. Ciambrone, ${ }^{19}$ X. Cid Vidal, ${ }^{39}$ G. Ciezarek, ${ }^{43}$ P. E. L. Clarke, ${ }^{52}$ M. Clemencic, ${ }^{40}$ H. V. Cliff, ${ }^{49}$ J. Closier, ${ }^{40}$ J. Cogan, ${ }^{6}$ E. Cogneras, ${ }^{5}$ V. Cogoni, ${ }^{16, \mathrm{~g}}$ L. Cojocariu, ${ }^{30}$ P. Collins, ${ }^{40}$ T. Colombo, ${ }^{40}$ A. Comerma-Montells, ${ }^{12}$ A. Contu, ${ }^{40}$ A. Cook, ${ }^{48}$ G. Coombs, ${ }^{40}$ S. Coquereau, ${ }^{38}$ G. Corti, ${ }^{40}$ M. Corvo,,${ }^{17, a}$ C. M. Costa Sobral,${ }^{50}$ B. Couturier, ${ }^{40}$ G. A. Cowan,${ }^{52}$ D. C. Craik, ${ }^{58}$ A. Crocombe, ${ }^{50}$ M. Cruz Torres, ${ }^{1}$ R. Currie, ${ }^{52}$ C. D'Ambrosio, ${ }^{40}$ F. Da Cunha Marinho, ${ }^{2}$ E. Dall'Occo, ${ }^{43}$ J. Dalseno, ${ }^{48}$ A. Davis, ${ }^{3}$ O. De Aguiar Francisco, ${ }^{54}$ S. De Capua,${ }^{56}$ M. De Cian, ${ }^{12}$ J. M. De Miranda, ${ }^{1}$ L. De Paula, ${ }^{2}$ M. De Serio, ${ }^{14,1}$ P. De Simone, ${ }^{19}$ C. T. Dean, ${ }^{53}$ D. Decamp, ${ }^{4}$ L. Del Buono, ${ }^{8}$ H.-P. Dembinski, ${ }^{11}$ M. Demmer, ${ }^{10}$ A. Dendek, ${ }^{28}$ D. Derkach,${ }^{35}$ O. Deschamps, ${ }^{5}$ F. Dettori, ${ }^{54}$ B. Dey, ${ }^{65}$ A. Di Canto, ${ }^{40}$ P. Di Nezza, ${ }^{19}$ H. Dijkstra, ${ }^{40}$ F. Dordei, ${ }^{40}$ M. Dorigo,${ }^{41}$

A. Dosil Suárez, ${ }^{39}$ L. Douglas, ${ }^{53}$ A. Dovbnya ${ }^{45}$ K. Dreimanis, ${ }^{54}$ L. Dufour, ${ }^{43}$ G. Dujany, ${ }^{8}$ P. Durante,${ }^{40}$ R. Dzhelyadin, ${ }^{37}$ M. Dziewiecki, ${ }^{12}$ A. Dziurda, ${ }^{40}$ A. Dzyuba, ${ }^{31}$ S. Easo,${ }^{51}$ M. Ebert, ${ }^{52}$ U. Egede, ${ }^{55}$ V. Egorychev, ${ }^{32}$ S. Eidelman, ${ }^{36, f}$

S. Eisenhardt ${ }^{52}$ U. Eitschberger, ${ }^{10}$ R. Ekelhof,${ }^{10}$ L. Eklund, ${ }^{53}$ S. Ely, ${ }^{61}$ S. Esen, ${ }^{12}$ H. M. Evans,${ }^{49}$ T. Evans, ${ }^{57}$ A. Falabella, ${ }^{15}$ N. Farley, ${ }^{47}$ S. Farry,${ }^{54}$ R. Fay, ${ }^{54}$ D. Fazzini, ${ }^{21, d}$ L. Federici, ${ }^{25}$ D. Ferguson, ${ }^{52}$ G. Fernandez, ${ }^{38}$ P. Fernandez Declara, ${ }^{40}$ 
A. Fernandez Prieto ${ }^{39}$ F. Ferrari, ${ }^{15}$ F. Ferreira Rodrigues, ${ }^{2}$ M. Ferro-Luzzi, ${ }^{40}$ S. Filippov, ${ }^{34}$ R. A. Fini, ${ }^{14}$ M. Fiore,${ }^{17, a}$ M. Fiorini, ${ }^{17, a}$ M. Firlej ${ }^{28}$ C. Fitzpatrick,${ }^{41}$ T. Fiutowski, ${ }^{28}$ F. Fleuret,,${ }^{7, c}$ K. Fohll, ${ }^{40}$ M. Fontana,${ }^{16,40}$ F. Fontanelli, ${ }^{20, j}$ D. C. Forshaw,${ }^{61}$ R. Forty, ${ }^{40}$ V. Franco Lima,${ }^{54}$ M. Frank,${ }^{40}$ C. Frei,${ }^{40}$ J. Fu, ${ }^{22, \mathrm{~m}}$ W. Funk, ${ }^{40}$ E. Furfaro, ${ }^{25, \mathrm{i}}$ C. Färber, ${ }^{40}$ E. Gabriel,${ }^{52}$ A. Gallas Torreira, ${ }^{39}$ D. Galli,${ }^{15, h}$ S. Gallorini, ${ }^{23}$ S. Gambetta, ${ }^{52}$ M. Gandelman, ${ }^{2}$ P. Gandini,${ }^{57}$ Y. Gao, ${ }^{3}$ L. M. Garcia Martin, ${ }^{70}$ J. García Pardiñas, ${ }^{39}$ J. Garra Tico, ${ }^{49}$ L. Garrido, ${ }^{38}$ P. J. Garsed, ${ }^{49}$ D. Gascon, ${ }^{38}$ C. Gaspar, ${ }^{40}$ L. Gavardi, ${ }^{10}$ G. Gazzoni, ${ }^{5}$ D. Gerick, ${ }^{12}$ E. Gersabeck, ${ }^{12}$ M. Gersabeck, ${ }^{56}$ T. Gershon,${ }^{50}$ Ph. Ghez, ${ }^{4}$ S. Gianì, ${ }^{41}$ V. Gibson, ${ }^{49}$ O. G. Girard, ${ }^{41}$ L. Giubega,${ }^{30}$ K. Gizdov, ${ }^{52}$ V. V. Gligorov, ${ }^{8}$ D. Golubkov, ${ }^{32}$ A. Golutvin, ${ }^{55,40}$ A. Gomes, ${ }^{1, n}$ I. V. Gorelov, ${ }^{33}$ C. Gotti, ${ }^{21, d}$ E. Govorkova, ${ }^{43}$ J. P. Grabowski, ${ }^{12}$ R. Graciani Diaz,${ }^{38}$ L. A. Granado Cardoso, ${ }^{40}$ E. Graugés,${ }^{38}$ E. Graverini ${ }^{42}$ G. Graziani, ${ }^{18}$ A. Grecu, ${ }^{30}$ R. Greim, ${ }^{9}$ P. Griffith, ${ }^{16}$ L. Grillo, ${ }^{21,40, d}$ L. Gruber, ${ }^{40}$ B. R. Gruberg Cazon, ${ }^{57}$ O. Grünberg, ${ }^{67}$ E. Gushchin, ${ }^{34}$ Yu. Guz,${ }^{37}$ T. Gys, ${ }^{40}$ C. Göbel, ${ }^{62}$ T. Hadavizadeh, ${ }^{57}$ C. Hadjivasiliou, ${ }^{5}$ G. Haefeli, ${ }^{41}$ C. Haen, ${ }^{40}$ S. C. Haines, ${ }^{49}$ B. Hamilton, ${ }^{60}$ X. Han, ${ }^{12}$ T. H. Hancock, ${ }^{57}$ S. Hansmann-Menzemer, ${ }^{12}$ N. Harnew, ${ }^{57}$ S. T. Harnew, ${ }^{48}$ J. Harrison, ${ }^{56}$ C. Hasse,${ }^{40}$ M. Hatch, ${ }^{40}$ J. He, ${ }^{63}$ M. Hecker, ${ }^{55}$ K. Heinicke, ${ }^{10}$ A. Heister, ${ }^{9}$ K. Hennessy, ${ }^{54}$ P. Henrard, ${ }^{5}$ L. Henry, ${ }^{70}$ E. van Herwijnen, ${ }^{40}$ M. He $\beta,{ }^{67}$ A. Hicheur, ${ }^{2}$ D. Hill, ${ }^{57}$ C. Hombach,${ }^{56}$ P. H. Hopchev, ${ }^{41}$ Z.-C. Huard ${ }^{59}$ W. Hulsbergen, ${ }^{43}$ T. Humair, ${ }^{55}$ M. Hushchyn, ${ }^{35}$ D. Hutchcroft, ${ }^{54}$ P. Ibis, ${ }^{10}$ M. Idzik, ${ }^{28}$ P. Ilten, ${ }^{58}$ R. Jacobsson, ${ }^{40}$ J. Jalocha,${ }^{57}$ E. Jans, ${ }^{43}$ A. Jawahery, ${ }^{60}$ F. Jiang, ${ }^{3}$ M. John, ${ }^{57}$ D. Johnson, ${ }^{40}$ C. R. Jones, ${ }^{49}$ C. Joram, ${ }^{40}$ B. Jost, ${ }^{40}$ N. Jurik,${ }^{57}$ S. Kandybei, ${ }^{45}$ M. Karacson, ${ }^{40}$ J. M. Kariuki, ${ }^{48}$ S. Karodia, ${ }^{53}$ N. Kazeev, ${ }^{35}$ M. Kecke, ${ }^{12}$ M. Kelsey,${ }^{61}$ M. Kenzie, ${ }^{49}$ T. Ketel, ${ }^{44}$ E. Khairullin, ${ }^{35}$ B. Khanji, ${ }^{12}$ C. Khurewathanakul, ${ }^{41}$ T. Kirn, ${ }^{9}$ S. Klaver, ${ }^{56}$ K. Klimaszewski, ${ }^{29}$ T. Klimkovich, ${ }^{11}$ S. Koliiev, ${ }^{46}$ M. Kolpin, ${ }^{12}$ I. Komarov,${ }^{41}$ R. Kopecna, ${ }^{12}$ P. Koppenburg, ${ }^{43}$ A. Kosmyntseva, ${ }^{32}$ S. Kotriakhova, ${ }^{31}$ M. Kozeiha, ${ }^{5}$ M. Kreps,${ }^{50}$ P. Krokovny, ${ }^{36, \mathrm{f}}$ F. Kruse, ${ }^{10}$ W. Krzemien, ${ }^{29}$ W. Kucewicz, ${ }^{27,0}$ M. Kucharczyk,${ }^{27}$ V. Kudryavtsev, ${ }^{36, \mathrm{f}}$ A. K. Kuonen, ${ }^{41}$ K. Kurek, ${ }^{29}$ T. Kvaratskheliya, ${ }^{32,40}$ D. Lacarrere, ${ }^{40}$ G. Lafferty, ${ }^{56}$ A. Lai, ${ }^{16}$ G. Lanfranchi, ${ }^{19}$ C. Langenbruch, ${ }^{9}$ T. Latham, ${ }^{50}$ C. Lazzeroni, ${ }^{47}$ R. Le Gac, ${ }^{6}$ J. van Leerdam, ${ }^{43}$ A. Leflat, ${ }^{33,40}$ J. Lefrançois, ${ }^{7}$ R. Lefèvre, ${ }^{5}$ F. Lemaitre, ${ }^{40}$ E. Lemos Cid, ${ }^{39}$ O. Leroy, ${ }^{6}$ T. Lesiak, ${ }^{27}$ B. Leverington, ${ }^{12}$ P.-R. Li ${ }^{63}$ T. Li, ${ }^{3}$ Y. Li, ${ }^{7}$ Z. Li, ${ }^{61}$ T. Likhomanenko, ${ }^{68}$ R. Lindner, ${ }^{40}$ F. Lionetto, ${ }^{42}$ V. Lisovskyi, ${ }^{7}$ X. Liu, ${ }^{3}$ D. Loh ${ }^{50}$ A. Loi, ${ }^{16}$ I. Longstaff, ${ }^{53}$ J. H. Lopes, ${ }^{2}$ D. Lucchesi, ${ }^{23, p}$ M. Lucio Martinez, ${ }^{39}$ H. Luo,${ }^{52}$ A. Lupato, ${ }^{23}$ E. Luppi, ${ }^{17, a}$ O. Lupton, ${ }^{40}$ A. Lusiani, ${ }^{24}$ X. Lyu ${ }^{63}$ F. Machefert, ${ }^{7}$ F. Maciuc, ${ }^{30}$ V. Macko, ${ }^{41}$ P. Mackowiak,${ }^{10}$ S. Maddrell-Mander, ${ }^{48}$ O. Maev, ${ }^{31}$ K. Maguire,${ }^{56}$ D. Maisuzenko, ${ }^{31}$ M. W. Majewski, ${ }^{28}$ S. Malde, ${ }^{57}$ A. Malinin, ${ }^{68}$ T. Maltsev, ${ }^{36, \mathrm{f}}$ G. Manca, ${ }^{16, \mathrm{~g}}$ G. Mancinelli, ${ }^{6}$ P. Manning, ${ }^{61}$ D. Marangotto, ${ }^{22, \mathrm{~m}}$ J. Maratas, ${ }^{5, \mathrm{q}}$ J. F. Marchand, ${ }^{4}$ U. Marconi, ${ }^{15}$ C. Marin Benito, ${ }^{38}$ M. Marinangeli, ${ }^{41}$ P. Marino, ${ }^{41}$ J. Marks, ${ }^{12}$ G. Martellotti, ${ }^{26}$ M. Martin, ${ }^{6}$ M. Martinelli, ${ }^{41}$

D. Martinez Santos, ${ }^{39}$ F. Martinez Vidal,${ }^{70}$ D. Martins Tostes, ${ }^{2}$ L. M. Massacrier, ${ }^{7}$ A. Massafferri, ${ }^{1}$ R. Matev, ${ }^{40}$ A. Mathad ${ }^{50}$ Z. Mathe ${ }^{40}$ C. Matteuzzi, ${ }^{21}$ A. Mauri, ${ }^{42}$ E. Maurice, ${ }^{7, c}$ B. Maurin, ${ }^{41}$ A. Mazurov ${ }^{47}$ M. McCann,${ }^{55,40}$ A. McNab, ${ }^{56}$ R. McNulty, ${ }^{13}$ J. V. Mead, ${ }^{54}$ B. Meadows,${ }^{59}$ C. Meaux,${ }^{6}$ F. Meier, ${ }^{10}$ N. Meinert, ${ }^{67}$ D. Melnychuk, ${ }^{29}$ M. Merk, ${ }^{43}$ A. Merli, ${ }^{22,40, m}$ E. Michielin, ${ }^{23}$ D. A. Milanes, ${ }^{66}$ E. Millard, ${ }^{50}$ M.-N. Minard, ${ }^{4}$ L. Minzoni, ${ }^{17}$ D. S. Mitzel, ${ }^{12}$ A. Mogini, ${ }^{8}$ J. Molina Rodriguez, ${ }^{1}$ T. Mombacher, ${ }^{10}$ I. A. Monroy, ${ }^{66}$ S. Monteil,${ }^{5}$ M. Morandin, ${ }^{23}$ M. J. Morello, ${ }^{24, k}$ O. Morgunova, ${ }^{68}$ J. Moron, ${ }^{28}$ A. B. Morris, ${ }^{52}$ R. Mountain, ${ }^{61}$ F. Muheim, ${ }^{52}$ M. Mulder, ${ }^{43}$ D. Müller, ${ }^{56}$ J. Müller, ${ }^{10}$ K. Müller, ${ }^{42}$ V. Müller,${ }^{10}$ P. Naik, ${ }^{48}$ T. Nakada, ${ }^{41}$ R. Nandakumar, ${ }^{51}$ A. Nandi,${ }^{57}$ I. Nasteva, ${ }^{2}$ M. Needham,${ }^{52}$ N. Neri, ${ }^{22,40}$ S. Neubert, ${ }^{12}$ N. Neufeld,${ }^{40}$ M. Neuner, ${ }^{12}$ T. D. Nguyen, ${ }^{41}$ C. Nguyen-Mau, ${ }^{41, r}$ S. Nieswand,,${ }^{9}$ R. Niet, ${ }^{10}$ N. Nikitin, ${ }^{33}$ T. Nikodem, ${ }^{12}$ A. Nogay, ${ }^{68}$ D. P. O’Hanlon, ${ }^{50}$ A. Oblakowska-Mucha, ${ }^{28}$ V. Obraztsov, ${ }^{37}$ S. Ogilvy, ${ }^{19}$ R. Oldeman, ${ }^{16,9}$ C. J. G. Onderwater, ${ }^{71}$

A. Ossowska, ${ }^{27}$ J. M. Otalora Goicochea, ${ }^{2}$ P. Owen, ${ }^{42}$ A. Oyanguren,${ }^{70}$ P. R. Pais, ${ }^{41}$ A. Palano, ${ }^{14,1}$ M. Palutan,,${ }^{19,40}$ A. Papanestis ${ }^{51}$ M. Pappagallo, ${ }^{14,1}$ L. L. Pappalardo,${ }^{17, a}$ W. Parker ${ }^{60}$ C. Parkes,${ }^{56}$ G. Passaleva, ${ }^{18}$ A. Pastore, ${ }^{14,1}$ M. Patel,${ }^{55}$ C. Patrignani ${ }^{15, h}$ A. Pearce,${ }^{40}$ A. Pellegrino,${ }^{43}$ G. Penso, ${ }^{26}$ M. Pepe Altarelli ${ }^{40}$ S. Perazzini,${ }^{40}$ P. Perret,${ }^{5}$ L. Pescatore,${ }^{41}$ K. Petridis, ${ }^{48}$ A. Petrolini, ${ }^{20, j}$ A. Petrov,${ }^{68}$ M. Petruzzo, ${ }^{22, m}$ E. Picatoste Olloqui, ${ }^{38}$ B. Pietrzyk,,${ }^{4}$ M. Pikies,${ }^{27}$ D. Pinci, ${ }^{26}$ A. Pistone,${ }^{20, j}$ A. Piucci, ${ }^{12}$ V. Placinta, ${ }^{30}$ S. Playfer, ${ }^{52}$ M. Plo Casasus,${ }^{39}$ F. Polci, ${ }^{8}$ M. Poli Lener, ${ }^{19}$ A. Poluektov, ${ }^{50,36}$ I. Polyakov, ${ }^{61}$ E. Polycarpo, ${ }^{2}$ G. J. Pomery ${ }^{48}$ S. Ponce,${ }^{40}$ A. Popov,${ }^{37}$ D. Popov, ${ }^{11,40}$ S. Poslavskii, ${ }^{37}$ C. Potterat, ${ }^{2}$ E. Price,${ }^{48}$ J. Prisciandaro, ${ }^{39}$ C. Prouve, ${ }^{48}$ V. Pugatch, ${ }^{46}$ A. Puig Navarro, ${ }^{42}$ H. Pullen, ${ }^{57}$ G. Punzi, ${ }^{24, s}$ W. Qian, ${ }^{50}$ R. Quagliani, ${ }^{7,48}$ B. Quintana, ${ }^{5}$ B. Rachwal, ${ }^{28}$ J. H. Rademacker, ${ }^{48}$ M. Rama, ${ }^{24}$ M. Ramos Pernas,${ }^{39}$ M. S. Rangel, ${ }^{2}$ I. Raniuk,${ }^{45}$ F. Ratnikov, ${ }^{35}$ G. Raven, ${ }^{44}$ M. Ravonel Salzgeber ${ }^{40}$ M. Reboud, ${ }^{4}$ F. Redi, ${ }^{55}$ S. Reichert, ${ }^{10}$ A. C. dos Reis, ${ }^{1}$ C. Remon Alepuz, ${ }^{70}$ V. Renaudin, ${ }^{7}$ S. Ricciardi, ${ }^{51}$ S. Richards, ${ }^{48}$ M. Rihl,${ }^{40}$ K. Rinnert, ${ }^{54}$ V. Rives Molina,${ }^{38}$ P. Robbe, ${ }^{7}$ A. Robert, ${ }^{8}$ A. B. Rodrigues, ${ }^{1}$ E. Rodrigues,${ }^{59}$ J. A. Rodriguez Lopez,${ }^{66}$ P. Rodriguez Perez,${ }^{56}$ A. Rogozhnikov,${ }^{35}$ S. Roiser, ${ }^{40}$ A. Rollings ${ }^{57}$ V. Romanovskiy, ${ }^{37}$ A. Romero Vidal, ${ }^{39}$ J. W. Ronayne, ${ }^{13}$ M. Rotondo, ${ }^{19}$ M. S. Rudolph, ${ }^{61}$ T. Ruf, ${ }^{40}$ P. Ruiz Valls, ${ }^{70}$ J. Ruiz Vidal, ${ }^{70}$ J. J. Saborido Silva,${ }^{39}$ E. Sadykhov, ${ }^{32}$ N. Sagidova, ${ }^{31}$ B. Saitta, ${ }^{16, \mathrm{~g}}$ V. Salustino Guimaraes, ${ }^{1}$ 
C. Sanchez Mayordomo, ${ }^{70}$ B. Sanmartin Sedes, ${ }^{39}$ R. Santacesaria, ${ }^{26}$ C. Santamarina Rios, ${ }^{39}$ M. Santimaria,${ }^{19}$ E. Santovetti, ${ }^{25, \mathrm{i}}$ G. Sarpis, ${ }^{56}$ A. Sarti, ${ }^{26}$ C. Satriano, ${ }^{26, t}$ A. Satta, ${ }^{25}$ D. M. Saunders, ${ }^{48}$ D. Savrina, ${ }^{32,33}$ S. Schael, ${ }^{9}$ M. Schellenberg, ${ }^{10}$ M. Schiller, ${ }^{53}$ H. Schindler, ${ }^{40}$ M. Schlupp,${ }^{10}$ M. Schmelling, ${ }^{11}$ T. Schmelzer,${ }^{10}$ B. Schmidt,${ }^{40}$ O. Schneider, ${ }^{41}$ A. Schopper, ${ }^{40}$ H. F. Schreiner, ${ }^{59}$ K. Schubert, ${ }^{10}$ M. Schubiger, ${ }^{41}$ M.-H. Schune, ${ }^{7}$ R. Schwemmer, ${ }^{40}$ B. Sciascia, ${ }^{19}$ A. Sciubba, ${ }^{26, u}$ A. Semennikov, ${ }^{32}$ A. Sergi, ${ }^{47}$ N. Serra, ${ }^{42}$ J. Serrano, ${ }^{6}$ L. Sestini, ${ }^{23}$ P. Seyfert, ${ }^{40}$ M. Shapkin,${ }^{37}$ I. Shapoval, ${ }^{45}$ Y. Shcheglov, ${ }^{31}$ T. Shears, ${ }^{54}$ L. Shekhtman,${ }^{36, f}$ V. Shevchenko, ${ }^{68}$ B. G. Siddi,,${ }^{17,40}$ R. Silva Coutinho, ${ }^{42}$ L. Silva de Oliveira, ${ }^{2}$ G. Simi, ${ }^{23, p}$ S. Simone, ${ }^{14,1}$ M. Sirendi, ${ }^{49}$ N. Skidmore, ${ }^{48}$ T. Skwarnicki, ${ }^{61}$ E. Smith ${ }^{55}$ I. T. Smith, ${ }^{52}$ J. Smith, ${ }^{49}$ M. Smith, ${ }^{55}$ 1. Soares Lavra, ${ }^{1}$ M. D. Sokoloff, ${ }^{59}$ F. J. P. Soler, ${ }^{53}$ B. Souza De Paula, ${ }^{2}$ B. Spaan, ${ }^{10}$ P. Spradlin, ${ }^{53}$ S. Sridharan, ${ }^{40}$ F. Stagni, ${ }^{40}$ M. Stahl, ${ }^{12}$ S. Stahl, ${ }^{40}$ P. Stefko, ${ }^{41}$ S. Stefkova, ${ }^{55}$ O. Steinkamp, ${ }^{42}$ S. Stemmle, ${ }^{12}$ O. Stenyakin, ${ }^{37}$ M. Stepanova, ${ }^{31}$ H. Stevens, ${ }^{10}$ S. Stone, ${ }^{61}$ B. Storaci, ${ }^{42}$ S. Stracka, ${ }^{24, s}$ M. E. Stramaglia, ${ }^{41}$ M. Straticiuc, ${ }^{30}$ U. Straumann, ${ }^{42}$ L. Sun, ${ }^{64}$ W. Sutcliffe, ${ }^{55}$ K. Swientek, ${ }^{28}$ V. Syropoulos, ${ }^{44}$ M. Szczekowski, ${ }^{29}$ T. Szumlak, ${ }^{28}$ M. Szymanski, ${ }^{63}$ S. T' Jampens, ${ }^{4}$ A. Tayduganov, ${ }^{6}$ T. Tekampe, ${ }^{10}$ G. Tellarini, ${ }^{17, a}$ F. Teubert, ${ }^{40}$ E. Thomas, ${ }^{40}$ J. van Tilburg, ${ }^{43}$ M. J. Tilley, ${ }^{55}$ V. Tisserand, ${ }^{4}$ M. Tobin, ${ }^{41}$ S. Tolk,${ }^{49}$ L. Tomassetti, ${ }^{17, a}$ D. Tonelli, ${ }^{24}$ F. Toriello, ${ }^{61}$ R. Tourinho Jadallah Aoude, ${ }^{1}$ E. Tournefier,${ }^{4}$ M. Traill,${ }^{53}$ M. T. Tran, ${ }^{41}$ M. Tresch, ${ }^{42}$ A. Trisovic,${ }^{40}$ A. Tsaregorodtsev, ${ }^{6}$ P. Tsopelas, ${ }^{43}$ A. Tully, ${ }^{49}$ N. Tuning, ${ }^{43}$ A. Ukleja, ${ }^{29}$ A. Usachov, ${ }^{7}$ A. Ustyuzhanin, ${ }^{35}$ U. Uwer, ${ }^{12}$ C. Vacca, ${ }^{16,9}$ A. Vagner, ${ }^{69}$ V. Vagnoni, ${ }^{15,40}$ A. Valassi, ${ }^{40}$ S. Valat,${ }^{40}$ G. Valenti, ${ }^{15}$ R. Vazquez Gomez, ${ }^{19}$ P. Vazquez Regueiro, ${ }^{39}$ S. Vecchi, ${ }^{17}$ M. van Veghel, ${ }^{43}$ J. J. Velthuis, ${ }^{48}$ M. Veltri, ${ }^{18, v}$ G. Veneziano, ${ }^{57}$ A. Venkateswaran, ${ }^{61}$ T. A. Verlage, ${ }^{9}$ M. Vernet,${ }^{5}$ M. Vesterinen, ${ }^{57}$ J. V. Viana Barbosa, ${ }^{40}$ B. Viaud, ${ }^{7}$ D. Vieira, ${ }^{63}$ M. Vieites Diaz, ${ }^{39}$ H. Viemann, ${ }^{67}$ X. Vilasis-Cardona,${ }^{38, b}$ M. Vitti, ${ }^{49}$ V. Volkov,${ }^{33}$ A. Vollhardt, ${ }^{42}$ B. Voneki, ${ }^{40}$ A. Vorobyev,${ }^{31}$ V. Vorobyev, ${ }^{36, f}$ C. Voß,${ }^{9}$ J. A. de Vries, ${ }^{43}$ C. Vázquez Sierra ${ }^{39}$ R. Waldi, ${ }^{67}$ C. Wallace,${ }^{50}$ R. Wallace, ${ }^{13}$ J. Walsh,${ }^{24}$ J. Wang,${ }^{61}$ D. R. Ward ${ }^{49}$ H. M. Wark, ${ }^{54}$ N. K. Watson, ${ }^{47}$ D. Websdale, ${ }^{55}$ A. Weiden, ${ }^{42}$ M. Whitehead, ${ }^{40}$ J. Wicht, ${ }^{50}$ G. Wilkinson, ${ }^{57,40}$ M. Wilkinson, ${ }^{61} \mathrm{M}$. Williams, ${ }^{56} \mathrm{M}$. P. Williams, ${ }^{47} \mathrm{M}$. Williams, ${ }^{58} \mathrm{~T}$. Williams, ${ }^{47}$ F. F. Wilson, ${ }^{51} \mathrm{~J}$. Wimberley, ${ }^{60}$ M. A. Winn, ${ }^{7}$ J. Wishahi, ${ }^{10}$ W. Wislicki,${ }^{29}$ M. Witek, ${ }^{27}$ G. Wormser, ${ }^{7}$ S. A. Wotton, ${ }^{49}$ K. Wraight,${ }^{53}$ K. Wyllie, ${ }^{40}$ Y. Xie, ${ }^{65}$ Z. Xu, ${ }^{4}$ Z. Yang, ${ }^{3}$ Z. Yang, ${ }^{60}$ Y. Yao, ${ }^{61}$ H. Yin ${ }^{65}$ J. Yu,${ }^{65}$ X. Yuan,${ }^{61}$ O. Yushchenko, ${ }^{37}$ K. A. Zarebski, ${ }^{47}$ M. Zavertyaev, ${ }^{11, w}$ L. Zhang, ${ }^{3}$ Y. Zhang, ${ }^{7}$ A. Zhelezov, ${ }^{12}$ Y. Zheng, ${ }^{63}$ X. Zhu, ${ }^{3}$ V. Zhukov, ${ }^{33}$ J. B. Zonneveld, ${ }^{52}$ and S. Zucchelli ${ }^{15}$

\title{
(LHCb Collaboration)
}

\author{
${ }^{1}$ Centro Brasileiro de Pesquisas Físicas (CBPF), Rio de Janeiro, Brazil \\ ${ }^{2}$ Universidade Federal do Rio de Janeiro (UFRJ), Rio de Janeiro, Brazil \\ ${ }^{3}$ Center for High Energy Physics, Tsinghua University, Beijing, China \\ ${ }^{4} L A P P$, Université Savoie Mont-Blanc, CNRS/IN2P3, Annecy-Le-Vieux, France \\ ${ }^{5}$ Clermont Université, Université Blaise Pascal, CNRS/IN2P3, LPC, Clermont-Ferrand, France \\ ${ }^{6}$ CPPM, Aix-Marseille Université, CNRS/IN2P3, Marseille, France \\ ${ }^{7}$ LAL, Université Paris-Sud, CNRS/IN2P3, Orsay, France \\ ${ }^{8}$ LPNHE, Université Pierre et Marie Curie, Université Paris Diderot, CNRS/IN2P3, Paris, France \\ ${ }^{9}$ I. Physikalisches Institut, RWTH Aachen University, Aachen, Germany \\ ${ }^{10}$ Fakultät Physik, Technische Universität Dortmund, Dortmund, Germany \\ ${ }^{11}$ Max-Planck-Institut für Kernphysik (MPIK), Heidelberg, Germany \\ ${ }^{12}$ Physikalisches Institut, Ruprecht-Karls-Universität Heidelberg, Heidelberg, Germany \\ ${ }^{13}$ School of Physics, University College Dublin, Dublin, Ireland \\ ${ }^{14}$ Sezione INFN di Bari, Bari, Italy \\ ${ }^{15}$ Sezione INFN di Bologna, Bologna, Italy \\ ${ }^{16}$ Sezione INFN di Cagliari, Cagliari, Italy \\ ${ }^{17}$ Universita e INFN, Ferrara, Ferrara, Italy \\ ${ }^{18}$ Sezione INFN di Firenze, Firenze, Italy \\ ${ }^{19}$ Laboratori Nazionali dell'INFN di Frascati, Frascati, Italy \\ ${ }^{20}$ Sezione INFN di Genova, Genova, Italy \\ ${ }^{21}$ Universita e INFN, Milano-Bicocca, Milano, Italy \\ ${ }^{22}$ Sezione di Milano, Milano, Italy \\ ${ }^{23}$ Sezione INFN di Padova, Padova, Italy \\ ${ }^{24}$ Sezione INFN di Pisa, Pisa, Italy \\ ${ }^{25}$ Sezione INFN di Roma Tor Vergata, Roma, Italy \\ ${ }^{26}$ Sezione INFN di Roma La Sapienza, Roma, Italy
}


${ }^{27}$ Henryk Niewodniczanski Institute of Nuclear Physics Polish Academy of Sciences, Kraków, Poland

${ }^{28}$ AGH - University of Science and Technology, Faculty of Physics and Applied Computer Science, Kraków, Poland

${ }^{29}$ National Center for Nuclear Research (NCBJ), Warsaw, Poland

${ }^{30}$ Horia Hulubei National Institute of Physics and Nuclear Engineering, Bucharest-Magurele, Romania

${ }^{31}$ Petersburg Nuclear Physics Institute (PNPI), Gatchina, Russia

${ }^{32}$ Institute of Theoretical and Experimental Physics (ITEP), Moscow, Russia

${ }^{33}$ Institute of Nuclear Physics, Moscow State University (SINP MSU), Moscow, Russia

${ }^{34}$ Institute for Nuclear Research of the Russian Academy of Sciences (INR RAN), Moscow, Russia

${ }^{35}$ Yandex School of Data Analysis, Moscow, Russia

${ }^{36}$ Budker Institute of Nuclear Physics (SB RAS), Novosibirsk, Russia

${ }^{37}$ Institute for High Energy Physics (IHEP), Protvino, Russia

${ }^{38}$ ICCUB, Universitat de Barcelona, Barcelona, Spain

${ }^{39}$ Universidad de Santiago de Compostela, Santiago de Compostela, Spain

${ }^{40}$ European Organization for Nuclear Research (CERN), Geneva, Switzerland

${ }^{41}$ Institute of Physics, Ecole Polytechnique Fédérale de Lausanne (EPFL), Lausanne, Switzerland

${ }^{42}$ Physik-Institut, Universität Zürich, Zürich, Switzerland

${ }^{43}$ Nikhef National Institute for Subatomic Physics, Amsterdam, Netherlands

${ }^{44}$ Nikhef National Institute for Subatomic Physics and VU University Amsterdam, Amsterdam, Netherlands

${ }^{45}$ NSC Kharkiv Institute of Physics and Technology (NSC KIPT), Kharkiv, Ukraine

${ }^{46}$ Institute for Nuclear Research of the National Academy of Sciences (KINR), Kyiv, Ukraine

${ }^{47}$ University of Birmingham, Birmingham, United Kingdom

${ }^{48}$ H.H. Wills Physics Laboratory, University of Bristol, Bristol, United Kingdom

${ }^{49}$ Cavendish Laboratory, University of Cambridge, Cambridge, United Kingdom

${ }^{50}$ Department of Physics, University of Warwick, Coventry, United Kingdom

${ }^{51}$ STFC Rutherford Appleton Laboratory, Didcot, United Kingdom

${ }^{52}$ School of Physics and Astronomy, University of Edinburgh, Edinburgh, United Kingdom

${ }^{53}$ School of Physics and Astronomy, University of Glasgow, Glasgow, United Kingdom

${ }^{54}$ Oliver Lodge Laboratory, University of Liverpool, Liverpool, United Kingdom

${ }^{55}$ Imperial College London, London, United Kingdom

${ }^{56}$ School of Physics and Astronomy, University of Manchester, Manchester, United Kingdom

${ }^{57}$ Department of Physics, University of Oxford, Oxford, United Kingdom

${ }^{58}$ Massachusetts Institute of Technology, Cambridge, Massachusetts, USA

${ }^{59}$ University of Cincinnati, Cincinnati, Ohio, USA

${ }^{60}$ University of Maryland, College Park, Maryland, USA

${ }^{61}$ Syracuse University, Syracuse, New York, USA

${ }^{62}$ Pontifícia Universidade Católica do Rio de Janeiro (PUC-Rio), Rio de Janeiro, Brazil

(associated with Institution Universidade Federal do Rio de Janeiro (UFRJ), Rio de Janeiro, Brazil)

${ }^{63}$ University of Chinese Academy of Sciences, Beijing, China

(associated with Institution Center for High Energy Physics, Tsinghua University, Beijing, China)

${ }^{64}$ School of Physics and Technology, Wuhan University, Wuhan, China

(associated with Institution Center for High Energy Physics, Tsinghua University, Beijing, China)

${ }^{65}$ Institute of Particle Physics, Central China Normal University, Wuhan, Hubei, China

(associated with Institution Center for High Energy Physics, Tsinghua University, Beijing, China)

${ }^{66}$ Departamento de Fisica, Universidad Nacional de Colombia, Bogota, Colombia

(associated with Institution LPNHE, Université Pierre et Marie Curie, Université Paris Diderot, CNRS/IN2P3, Paris, France)

${ }^{67}$ Institut für Physik, Universität Rostock, Rostock, Germany

(associated with Institution Physikalisches Institut, Ruprecht-Karls-Universität Heidelberg, Heidelberg, Germany)

${ }^{68}$ National Research Centre Kurchatov Institute, Moscow, Russia

(associated with Institution Institute of Theoretical and Experimental Physics (ITEP), Moscow, Russia)

${ }^{69}$ National Research Tomsk Polytechnic University, Tomsk, Russia

(associated with Institution Institute of Theoretical and Experimental Physics (ITEP), Moscow, Russia)

${ }^{70}$ Instituto de Fisica Corpuscular, Centro Mixto Universidad de Valencia - CSIC, Valencia, Spain

(associated with Institution ICCUB, Universitat de Barcelona, Barcelona, Spain)

${ }^{71}$ Van Swinderen Institute, University of Groningen, Groningen, Netherlands

(associated with Institution Nikhef National Institute for Subatomic Physics, Amsterdam, Netherlands)

${ }^{a}$ Also at Università di Ferrara, Ferrara, Italy.

${ }^{\mathrm{b}}$ Also at LIFAELS, La Salle, Universitat Ramon Llull, Barcelona, Spain.

${ }^{\mathrm{c}}$ Also at Laboratoire Leprince-Ringuet, Palaiseau, France.

dAlso at Università di Milano Bicocca, Milano, Italy. 
${ }^{\mathrm{e}}$ Also at Università di Modena e Reggio Emilia, Modena, Italy.

${ }^{\mathrm{f}}$ Also at Novosibirsk State University, Novosibirsk, Russia.

${ }^{\mathrm{g}}$ Also at Università di Cagliari, Cagliari, Italy.

${ }^{\mathrm{h}}$ Also at Università di Bologna, Bologna, Italy.

${ }^{\mathrm{i}}$ Also at Università di Roma Tor Vergata, Roma, Italy.

${ }^{\mathrm{j}}$ Also at Università di Genova, Genova, Italy.

${ }^{\mathrm{k}}$ Also at Scuola Normale Superiore, Pisa, Italy.

${ }^{1}$ Also at Università di Bari, Bari, Italy.

${ }^{\mathrm{m}}$ Also at Università degli Studi di Milano, Milano, Italy.

${ }^{\mathrm{n}}$ Also at Universidade Federal do Triângulo Mineiro (UFTM), Uberaba-MG, Brazil.

${ }^{\circ}$ Also at AGH - University of Science and Technology, Faculty of Computer Science, Electronics and Telecommunications, Kraków, Poland.

${ }^{\mathrm{p}}$ Also at Università di Padova, Padova, Italy.

${ }^{\mathrm{q}}$ Also at Iligan Institute of Technology (IIT), Iligan, Philippines.

${ }^{\mathrm{r}}$ Also at Hanoi University of Science, Hanoi, Viet Nam.

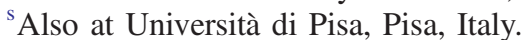

${ }^{\mathrm{t}}$ Also at Università della Basilicata, Potenza, Italy.

"Also at Università di Roma La Sapienza, Roma, Italy.

${ }^{v}$ Also at Università di Urbino, Urbino, Italy.

${ }^{\text {w}}$ Also at P.N. Lebedev Physical Institute, Russian Academy of Science (LPI RAS), Moscow, Russia. 